\title{
A Case Report on Physiotherapy Rehabilitation of Complex Regional Pain Syndrome (CRPS)
}

\author{
Priyanka Sahu, Ruchika zade, Gunjan shende ${ }^{1}$ and Kiran Kumar* \\ ${ }^{1}$ Department of Musculoskeletal Physiotherapy, Ravi Nair Physiotherapy College, \\ Datta Meghe Institute of Medical Sciences, Wardha, Maharashtra, India. \\ Corresponding author email: kiran.kumar@dmimsu.edu.in
}

\section{ABSTRACT}

Complex regional pain syndrome (CRPS) may develop subsequent to limb trauma and usually presents with sensory-motor and autonomic symptoms along with pain.Major CRPS symptoms include trauma-related cytokine release, exaggerated neurogenic inflammation, and neuroplasticity. The incidence of CRPS was estimated to be around 26.2 per 100,000 person years with male female ratio of 3.4. The highest incidence is reported in females aged 61-70 years. Patient with history of fall on right hand visited to private hospital. She had swelling for which plaster cast was applied for 7 days followed by permanent plaster cast applied for 21 days. After 21 days plaster cast was removed and patient was referred for physiotherapy. She complained of burning pain in the right hand for one and half months. X-ray confirmed fracture of distal end of radius. Features confirmed complex regional pain syndrome of the wrist. Specific treatment modalities included transcutaneous electrical nerve stimulation, progressive weight bearing, tactile desensitization, massage, and contrast baths. Patient recovered in 6 weeks therapy. Timely initiation of physical therapy is useful to reduce pain and swelling following fracture healing early recovery.

KEY WORDS: FRACTURE RADIUS, WRIST, CRPS, TENS, PULSED ELECTROMAGNETIC FIELD THERAPY, PARAFFIN WAX, TACTILE DESENSITIZATION, MASSAGE, AND CONTRAST BATHS.

\section{INTRODUCTION}

Complex regional pain syndrome (CRPS) was reported to develop followed by limb trauma. It presents with pain and sensory-motorautonomic symptoms. Most symptoms of CRPS, are trauma-related cytokine release, exaggerated neurogenic inflammation and neuroplasticity (Birklein et al., 2005). The overall incidence of CRPS was estimated to be around 26.2 per 100,000 person yearsand females were affected thrice compared to males (Ratio: 3.4 ). The highest incidence is seen in females aged 61-70 years. Upper limbs are affected frequently than lower limbs. Fracture seems to be a common precipitating event (44\%).

Biosc Biotech Res Comm P-ISSN: 0974-6455 E-ISSN: 2321-4007

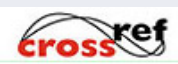

Identifiers and Pagination

Year: 2021 Vol: 14 No (6) Special Issue

Pages: 109-112

This is an open access article under Creative

Commons License Attribn 4.0 Intl (CC-BY).
DOI: $h t t p: / / d x . d o i . o r g / 10.21786 / b b r c / 14.25$

Commons License Attribn 4.0 Intl (CC-BY).
DOI: $h t t p: / / d x . d o i . o r g / 10.21786 / b b r c / 14.25$
Postmenopausal woman are affected more with CRPS(De Mos et al., 2007).

Comorbidities like diabetes, hyperlipidemia, gout and excessive alcohol consumptiondoes not show any association with CRPS. As per the study by Veldman (1995), administration of antiepileptic (phenytoin) and antituberculosis (isoniazid) drugs for longer duration increases the risk of RSD(Zyluk et al., 2004).

Patient information: Patient was apparently alright one and half month back during her household activity she fell down on her right hand then she immediately visited to private hospital, she had swelling for which plaster cast was applied for 7 days as the swelling subsided then permanent plaster cast was applied for 21 days after 21 days plaster cast was removed, medications were given and she was referred for physiotherapy. The pain aggravates on work and relieves on rest.

\section{Article Information}

Received: $30^{\text {th }}$ March 2021

ccepted after revision: $28^{\text {th }}$ May 2021 
Clinical Finding: Patient having complaint of burning pain in the right hand and swelling in both the hands since one and half month. On observation the body type is mesomorphic, gait appears to be normal, posture is normal, no shortening of limb. On examination active movement (Range of Motion) of wrist flexion, Extension, radial deviation and ulnar deviation is slightly restricted and painful at the end of range of motion. which is shown in table-1(before and after treatment range was taken). Manual Muscle Testing (MMT) was evaluated in available ROM, which is shown in table-2 (before treatment and after treatment grading was taken).

\section{Pain Assessment:}

Pre-rehab VAS: 8/10 at rest, 9/10 on slight movement. Post-rehab VAS: 6/10 at rest, 7/10 on slight movement.

Neurologic Examination was done where Dermatomes and myotomes was evaluated and there were no diminished or absent sensation, nerve roots were intact. Reflexes were intact.

Hand function assessment: Hand function were assessed for grasp and grip following findings were obtained.

Intervention: The medication were given and physical therapy also started, We took the patient's follow-up for 6 week of time.

\section{Description:}

Treatment

Physical Therapy: The physiotherapy treatment modalities included cognitive-behavioral therapy, transcutaneous electrical nerve stimulation, progressive weight bearing, tactile desensitization, massage, and contrast baths. All were instructed in home practice regimens.

Cognitive-Behavioural Therapy emphasized on problemsolving and coping with stressful life events. Audiotapes of relaxation techniques were provided for daily home use.

A standardized educational program was delivered which reviewed differences between nociceptive and neuropathic pain, differences between protective and non-protective pain. It also reflected on the importance of physical and psychosocial rehabilitation and the importance of active participation in treatment (Leeet al., 2002). Transcutaneous electrical nerve stimulation helped for improving pain (Albazaz et al., 2008). Manual drainage procedures and compression bandaging were applied during the physiotherapy sessions to alleviate swelling and minimize scar tissue production, and the patient was taught to hold the arm in an elevated position and to perform gentle active movements (Santacreu et al., 2016).

Table 1. Range of motion

\begin{tabular}{|l|c|c|c|c|}
\hline Movement & Pre-rehab active & Pre-rehab passive & Post-rehab active & Post-rehab passive \\
\hline Wrist flexion & $0-50$ & $0-55$ & $0-75$ & $0-80$ \\
\hline Wrist extension & $0-40$ & $0-45$ & $0-55$ & $0-60$ \\
\hline Ulnar deviation & $0-20$ & $0-25$ & $0-30$ & $0-35$ \\
\hline Radial deviation & $0-10$ & $0-10$ & $0-15$ & $0-15$ \\
\hline
\end{tabular}

Table 2. Manual Muscle testing (MMT)

\begin{tabular}{|l|c|c|}
\hline Movement & Pre-rehab & Post-rehab \\
\hline Wrist flexor & 3 & 4 \\
\hline Wrist extensor & 3 & 4 \\
\hline Wrist abductor & 4 & 4 \\
\hline Wrist adductor & 4 & 4 \\
\hline
\end{tabular}

Paraffin was applied for 20 min prior to manual therapy. Subjects received 6 weekly sessions of individual CBT incorporating pain management strategies (Devrimsel et al., 2015; Pagani et al., 2016;Mark et al., 2020),including relaxation training, deep breathing exercises, biofeedback, and guided imagery (10).Pulsed electromagnetic field therapy was used in association with calcitonin and stretching exercises (Pagani et al., 2016).

\begin{tabular}{|l|l|}
\hline Grasp a) spherical & No \\
\hline b) cylindrical & No \\
\hline c) hook & No \\
\hline Grip a) pulp to pulp & No \\
\hline b)tip to tip & No \\
\hline c) three jaw chunk & No \\
\hline d) lateral prehension & No \\
\hline
\end{tabular}

\section{RESULTS}

Patient found relief after 6 weeks of treatment.

Limitation: Patient took physiotherapy intervention for 6 weeks. The follow up sessions could not take place because there were no means of transport and her economic status was low hence, she was not able to take further treatment. 
Figure 1: post treatment in pulsed electromagnetic field therapy (PEMF)

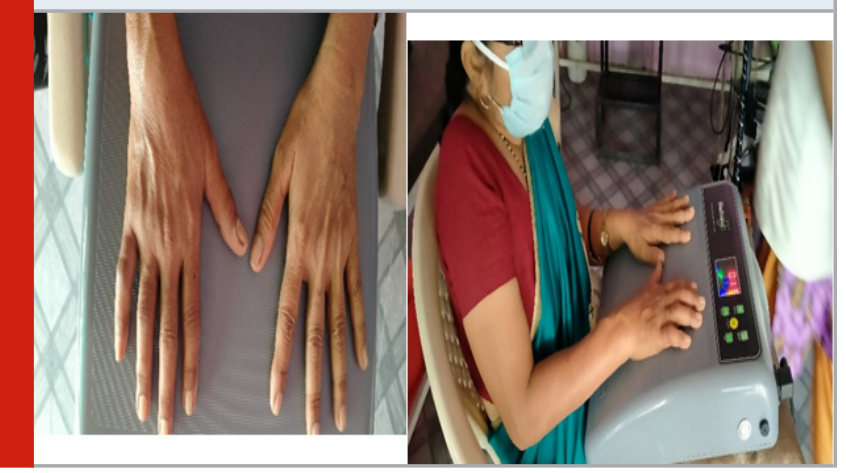

DISCUSSION

The precise CRPS prevalence is uncertain. Gender, Sex. There is no impact of race or geographical position on the incidence of CRPS. In general, in females and throughout the fourth decade of life, CRPS is more common. There is no big difference in the involvement of the dominant and the dominant Nondominant extremities, however presence of the upper extremities More frequent etiologies of fractures. CRPS-related discomfort improves the resistance of sufferers to moving the extremities, and joint stiffness tends to worsen. In patients, sudomotor and vasomotor modifications cause edema and variable skin discoloration.

CRPS, with. Since its physiopathology remains uncertain, CRPS is very hard to handle. As a consequence, considering the numerous therapeutic options available, an optimal therapy has not been determined. Another treatment choice for CRPS is neuromuscular electrical stimulation and it causes muscle contractions that compress the venous and lymphatic vessels. This mechanical impact helps to overcome edema and was used to resolve edema With considerable progress noted, treat hand edema noted. The present research, along with the literature found That both treatment regimens boosted the strength of the grip, Power of pinch (threepoint and fingertip pinch strengths), During care, and ROM of the wrist joints. These, however, Improvements in the whirlpool bath category were stronger.

The difference in lateral pinch intensity enhancement between the groups was not important. Studies from Global burden of disease were reviewed (Murrayet al., 2016; Murrayet al., 2019; Voset al., 2019;Wanget al., 2019;:Lozanoet al., 2020;Franklinet al., 2020). Studies and cases on physiotherapy rehabilitation of related condition were reported (Bawiskaret al., 2020;Phansopkaret al., 2020).

\section{CONCLUSION}

This case report provide patient with a comprehensive recovery that helped her to relieve pain and reduced swelling with increasing strength and range of motion.
Patient was able to perform basic activities of daily living.

\section{List of abbreviation}

TENS- transcutaneous electrical nerve stimulation

VAS - visual analogue scale

PEMF- pulsed electromagnetic field therapy

Authors contribution: All authors made best contribution for the concept, evaluation and assessment, interpretation of the data analysis and data acquisition.

\section{Conflict of interest: None}

\section{Funding support: None}

\section{REFERENCES}

Albazaz, R., Wong, Y.T. and Homer-Vanniasinkam, S., 2008. Complex regional pain syndrome: a review. Annals of vascular surgery, 22(2), pp.297-306.

Albazaz, R., Wong, Y.T. and Homer-Vanniasinkam, S., 2008. Complex regional pain syndrome: a review. Annals of vascular surgery, 22(2), pp.297-306.

Aldini, N.N. and Fini, M., 2017. Complex regional pain syndrome type I, a debilitating and poorly understood syndrome. Possible role for pulsed electromagnetic fields: a narrative review. Pain Physician, 20, pp.807E822.

Bawiskar, D.P., Bais, A.S., Naqvi, W.M. and Sahu, A., 2020. Physiotherapy Approach towards a Typical Case of Injection Induced Radial Nerve Palsy Presenting as Wrist Drop. Journal of Evolution of Medical and Dental Sciences, 9(22), pp.1722-1725.

Birklein F. 2005. Complex regional pain syndrome. J Neurol., 252(2),131-8.

de Mos, Marissa, A. G. J. De Bruijn, F. J. P. M. Huygen, J. P. Dieleman, BH ChStricker, and M. C. J. M. Sturkenboom. 2007. The incidence of complex regional pain syndrome: a population-based study. Pain, 129, 1-2 : 12-20.

Devrimsel, G., Turkyilmaz, A.K., Yildirim, M. and Beyazal, M.S., 2015. The effects of whirlpool bath and neuromuscular electrical stimulation on complex regional pain syndrome. Journal of physical therapy science, 27(1), pp.27-30.

Franklin, R.C., Peden, A.E., Hamilton, E.B., Bisignano, C., Castle, C.D., Dingels, Z.V., Hay, S.I., Liu, Z., Mokdad, A.H., Roberts, N.L. and Sylte, D.O., 2020. The burden of unintentional drowning: global, regional and national estimates of mortality from the Global Burden of Disease 2017 Study. Injury prevention, 26(Supp 1), pp.83-i95. Latchoumi, T.P., Ezhilarasi, T.P. and Balamurugan, K., 2019. Bio-inspired weighed quantum particle swarm optimization and smooth support vector machine ensembles for identification of abnormalities in medical data. SN Applied Sciences, 1(10), pp.1-10.

Lee, B.H., Scharff, L., Sethna, N.F., McCarthy, C.F., Scott-Sutherland, J., Shea, A.M., Sullivan, P., Meier, 
P., Zurakowski, D., Masek, B.J. and Berde, C.B., 2002. Physical therapy and cognitive-behavioral treatment for complex regional pain syndromes. The Journal of pediatrics, 141(1), pp.135-140.

Lozano, R., Fullman, N., Mumford, J.E., Knight, M., Barthelemy, C.M., Abbafati, C., Abbastabar, H., AbdAllah, F., Abdollahi, M., Abedi, A. and Abolhassani, H., 2020. Measuring universal health coverage based on an index of effective coverage of health services in 204 countries and territories, 1990-2019: a systematic analysis for the Global Burden of Disease Study 2019. The Lancet, 396(10258), pp.1250-1284.

Murray, C.J., Abbafati, C., Abbas, K.M., Abbasi, M., Abbasi-Kangevari, M., Abd-Allah, F., Abdollahi, M., Abedi, P., Abedi, A., Abolhassani, H. and Aboyans, V., 2020. Five insights from the global burden of disease study 2019. The Lancet, 396(10258), pp.1135-1159.

Murray, C.J., Aravkin, A.Y., Zheng, P., Abbafati, C., Abbas, K.M., Abbasi-Kangevari, M., Abd-Allah, F., Abdelalim, A., Abdollahi, M., Abdollahpour, I. and Abegaz, K.H., 2020. Global burden of 87 risk factors in 204 countries and territories, 1990-2019: a systematic analysis for the Global Burden of Disease Study 2019. The Lancet, 396(10258), pp.1223-1249.

Phansopkar, P., Athawale, V., Birelliwar, A., Naqvi, W. and Kamble, S., 2020. Post-operative rehabilitation in a traumatic rare radial nerve palsy managed with tendon transfers: a case report. The Pan African Medical Journal, 36.
Santacreu, E.S., Cabezas, N.V. and Graupera, A.B., 2016. Combined treatment with paraffin, manual therapy, pegboard and splinting in a patient with post-traumatic stiff hand. Archives of physiotherapy, 6(1), pp.1-6.

Shepherd, M., Louw, A. and Podolak, J., 2020. The clinical application of pain neuroscience, graded motor imagery, and graded activity with complex regional pain syndrome-A case report. Physiotherapy Theory and Practice, 36(9), pp.1043-1055.

Vos, T., Lim, S.S., Abbafati, C., Abbas, K.M., Abbasi, M., Abbasifard, M., Abbasi-Kangevari, M., Abbastabar, H., Abd-Allah, F., Abdelalim, A. and Abdollahi, M., 2020. Global burden of 369 diseases and injuries in 204 countries and territories, 1990-2019: a systematic analysis for the Global Burden of Disease Study 2019. The Lancet, 396(10258), pp.1204-1222.

Wang, H., Abbas, K.M., Abbasifard, M., AbbasiKangevari, M., Abbastabar, H., Abd-Allah, F., Abdelalim, A., Abolhassani, H., Abreu, L.G., Abrigo, M.R. and Abushouk, A.I., 2020. Global age-sex-specific fertility, mortality, healthy life expectancy (HALE), and population estimates in 204 countries and territories, 1950-2019: a comprehensive demographic analysis for the Global Burden of Disease Study 2019. The Lancet, 396(10258), pp.1160-1203.

Zyluk, A., 2004. Complex regional pain syndrome type I. Risk factors, prevention and risk of recurrence. Journal of Hand Surgery, 29(4), pp.334-337. 